\title{
La importancia del Trabajo Colaborativo en la enseñanza de la Metodología de Diseño de Proyectos.
}

The importance of collaborative work in teaching Project Design Methodology.

\author{
Mg. Arq. María Magdalena Peña \\ Fundación Universitaria Iberoamericana, Uruguay \\ tutor13.pry@funiber.org
}

\begin{abstract}
Nowadays organizations require their employees to develop transferable skills to take advantage in multidisciplinary teamworks.

In this context, it is important to implement within the teaching of Project Design Methodology at postgraduate level, some strategies that promote this type of competition through the creation of opportunities for collaborative work.

ICTs are presented as ideal tools for the implementation of teaching methods involving e-learning, and developing a suitable design of the interface with the student.

The advisor is the key and must act in every moment as a guide.
\end{abstract}

Keywords: Trabajo colaborativo; TICs; Proyectos.

\section{Contexto}

Cada vez más, a nivel del ejercicio de la actividad profesional, se tiende a funcionar con formas de trabajo que requieren de la colaboración interdisciplinaria.

Esta forma de cooperación requiere que los profesionales hayan desarrollado desde su etapa formativa de grado o de posgrado competencias y habilidades que la hagan posible y provechosa para sí mismos y para los resultados de la institución en la que se desempeñan.

Este tipo de gestión empresarial está basado en el diseño de espacios de intercambio virtuales que el desarrollo de las TICs hizo posible, pues permiten acentuar el aspecto relacional del trabajo anulando las dificultades impuestas por la distancia física de los colaboradores.

Cómo dijimos anteriormente, este modo de funcionamiento de las organizaciones, propio del siglo XXI, exige a los profesionales la posesión de competencias que denominamos transversales.

Siguiendo a Echazarreta, Prados, Poch y Soler (2009), podemos definir las competencias transversales o genéricas como aquellas que nacen de la intersección entre diferentes disciplinas.

En este contexto, una de las competencias transversales más valoradas en las más variadas áreas disciplinares es el trabajo colaborativo.

\section{Trabajo Colaborativo}

El trabajo colaborativo posee una serie de características propias que lo diferencian de otras modalidades de organización grupal:
- La fuerte interdependencia entre todos los miembros del equipo.

- La necesidad de la existencia de un compromiso de éxito del resultado final por parte de cada uno de los integrantes del conjunto.

- La heterogeneidad de habilidades personales en la conformación del grupo.

- $\quad$ El reconocimiento de responsabilidades individuales que deben ser, a su vez, compartidas por el colectivo.

- La interdependencia positiva entre los sujetos y

- La necesidad de desplegar habilidades comunicativas que permitan establecer relaciones simétricas y recíprocas a la interna del grupo que faciliten la consecución de un objetivo común.

\section{Estrategias Didácticas}

Desde la Academia, se hace menester dar una respuesta a estas necesidades asumiendo métodos de enseñanza y aprendizaje que hagan posible la adquisición y desarrollo, por parte de los estudiantes de grado y posgrado, de habilidades cognitivas y sociales, mediante la implementación de estrategias didácticas de trabajo grupal que tengan como premisa el acrecentamiento de las capacidades para establecer relaciones interpersonales entre los alumnos basadas en la interacción y la interdependencia y que estas competencias sean aprehendidas de forma tal que sean incorporadas más tarde al desarrollo de la actividad profesional de cada uno. 
Estas estrategias didácticas se orientan hacia un enfoque pedagógico centrado en el alumno y presentan determinadas características:

- Son metodologías didácticas que complejizan la labor del docente y requieren un alto grado de dedicación por la necesidad del diseño de material de apoyo y ayudas educativas.

- Las tareas planteadas al alumnado deben ser auténticas, relacionadas con las actividades profesionales de los miembros de cada grupo y responder a escenarios reales.

- Los problemas planteados deben ser abiertos y complejos para que los estudiantes puedan desarrollar soluciones creativas, originales, discutidas y consensuadas.

- Es necesario que los estudiantes laboren de manera relativamente autónoma durante un período de tiempo prolongado para arribar a un producto final satisfactorio.

\section{TICs}

Tal como dijimos anteriormente respecto a la gestión empresarial e institucional basada en el trabajo colaborativo, también, para la implementación de esta tipo de estrategias educativas, las TICs resultan sumamente apropiadas por tratarse de herramientas de comunicación sincrónicas y asincrónicas que facilitan las relaciones entre docente y los estudiantes y de estos últimos entre sí.

Si bien es posible la utilización de herramientas simples y abiertas (Wikis, Blogs, Foros, WebQuest, Chats), las plataformas educativas se muestran como la opción más idónea disponible por su diseño específico para cumplir este tipo de funciones.

Este tipo de plataformas proporcionan un entorno de aprendizaje que integra todas las herramientas informáticas necesarias para facilitar la participación activa del educando dentro de su proceso de aprendizaje, fomentando los espacios de colaboración, reflexión y crítica y conformando lo que denominamos un aula virtual.

Al plantearse una institución, como es el caso de FUNIBER, la aplicación de estas estrategias didácticas de aprendizaje colaborativo mediado por TICs, el paso previo es la elección de una plataforma educativa que sea capaz de proporcionar todas las herramientas informáticas necesarias para el desarrollo de las actividades curriculares.

Badía y García (2006) establecen una serie de instrumentos que una plataforma educativa que pretenda ser utilizada para la enseñanza y aprendizaje colaborativo de Metodología de Diseño de Proyectos debe ofrecer a estudiantes y profesores

En primer lugar, deben existir herramientas que permitan al docente, informar al alumnado sobre el proyecto pedagógico y el contenido de las actividades
En segundo tipo de instrumentos debe facilitar la comunicación entre el docente y cada uno de los estudiantes de manera particular, ya sea por cuestiones formativas, de contenido o administrativas.

En tercer término, son necesarias herramientas que posibiliten el intercambio entre el tutor y el equipo de trabajo, con la finalidad de evacuar dudas y, además, de que el docente pueda monitorear el desarrollo del proceso formativo

Por último, se requieren herramientas tecnológicas que ofrezcan la posibilidad de analizar, valorar y brindar un feedback a los estudiantes, por parte de la Tutoría respecto a cada una de las entregas parciales.

\section{Plataforma BSCW}

La plataforma escogida por FUNIBER para el desarrollo de su Módulo de Diseño de Proyectos es la BSCW, que proporciona, de manera satisfactoria, los instrumentos tecnológicos necesarios antes mencionados.

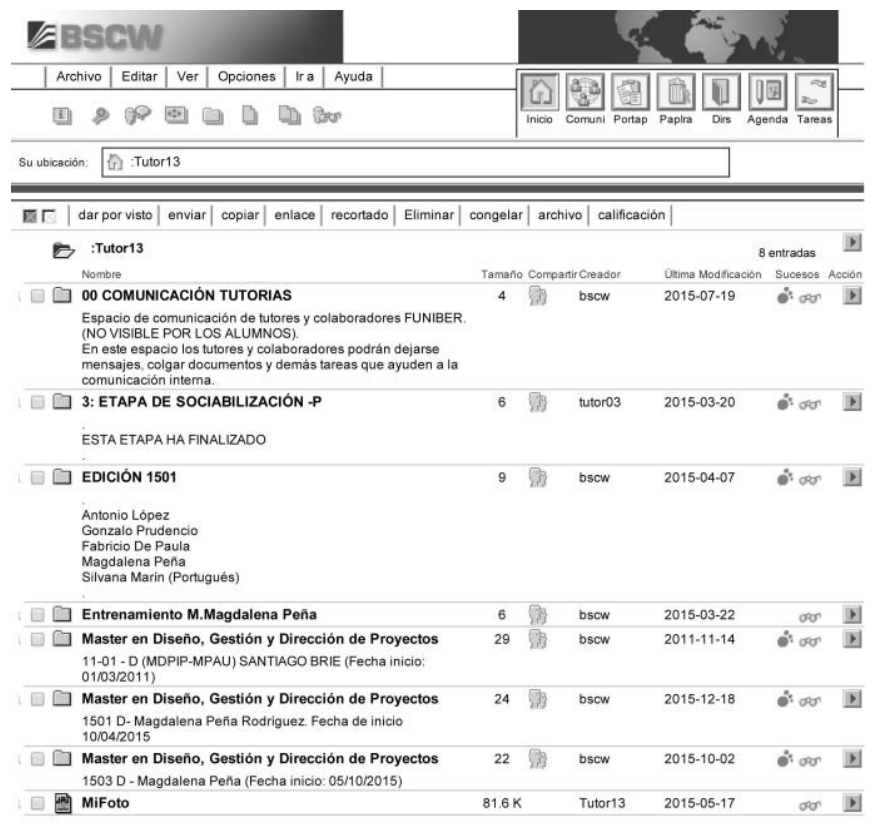

Figura 1: Acceso a la Plataforma BSCW. (Recuperada el 17 de febrero de 2016 de

http://bscw.ing.udep.edu.pe/bscw/bscw.cgi/8992013

En el caso particular de FUNIBER, las funciones docentes son desempeñadas de manera perfectamente coordinada por el Tutor y por la Coordinación Académica, los que mantienen entre sí fluida comunicación, por eso, de aquí en adelante nos referiremos a quienes cumplen este conjunto de tareas docentes como al Equipo de Tutorías o, simplemente, la Tutoría.

Más allá del método convencional, externo a la BSCW, que representa el correo electrónico y que en ocasiones es utilizado para comunicaciones puntuales, sobre todo de 
orden administrativo, la plataforma educativa escogida contiene herramientas comunicacionales apropiadas a la aplicación de una metodología de trabajo colaborativo.

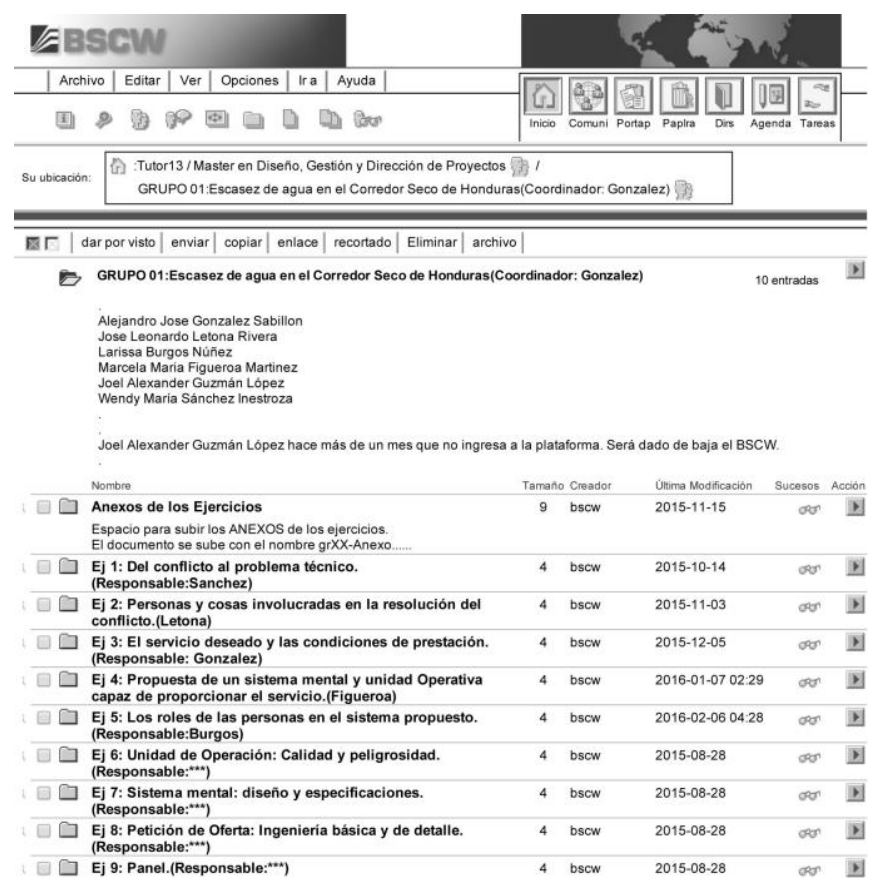

Figura 2: Carpeta de un equipo (Recuperada el 17 de febrero de 2016 de http://bscw.ing.udep.edu.pe/bscw/bscw.cgi/9700623)

La información acerca del desarrollo del módulo, calendario y contenidos teóricos, así como guías de ejecución y ejercitación, se agrupan en una carpeta específica, a la que todos los estudiantes tienen acceso, nombrada "Desarrollo del Proyecto".

Para las comunicaciones internas de cada grupo y del grupo con la Tutoría, cada uno de los equipos cuenta con una carpeta propia en la que pueden compartir información pertinente, trabajos individuales y colectivos y recibir las correcciones y sugerencias del Tutor.

Para cada una de las actividades parciales o ejercicios queda habilitado un "Foro de Debate del Ejercicio", que permite compartir inquietudes con la totalidad de los equipos y con la Tutoría.

\section{Metodología de Enseñanza de Diseño de Proyectos}

Al diseñar una metodología de enseñanza - aprendizaje colaborativo de Diseño de Proyectos del tipo de la implementada por FUNIBER, debe tenerse en cuenta, siguiendo los conceptos expresados por Badía y García (2006) que la misma debe contemplar una serie de etapas:

Desde un principio debe quedar establecido que se trabajará sobre problemas reales, relacionados con la actividad profesional de los alumnos.
Esta es una premisa fundamental que define el trabajo colaborativo y que no debe perderse de vista en ningún momento.

La conformación de los equipos de trabajo es clave para el éxito de la experiencia colaborativa.

Dos son los criterios que pueden adoptarse al respecto: el de la conformación de grupos heterogéneos, que tienden a obtener mejores resultados académicos pero es más factible que presenten problemas de gestión y comunicación o, por el contrario, la opción de trabajar con grupos homogéneos en los que las comunicaciones tienden a ser más fluidas pero el resultado es de menor calidad formativa.

El criterio de agrupación de FUNIBER puede decirse que es mixto: se trata de grupos heterogéneos en cuanto a sus orígenes geográficos y disciplinares y homogéneos en cuanto a sus intereses.

Antes de iniciar la ejercitación se habilita el foro "Etapa de Socialización" en el que los estudiantes se presentan a sí mismo y expresan sus intereses proyectuales, de manera que, espontánea y voluntariamente, se va produciendo la unión entre quienes tienen inquietudes similares.

La metodología de trabajo debe ser especificada dejando en claro objetivos, cronograma, formas de presentación y vías de comunicación.

Toda esta información se encuentra en las carpetas denominadas "Material Introductorio de Lectura Obligatoria" y "Desarrollo del Proyecto".

Deben establecerse y adoptarse normas claras de funcionamiento interno del grupo. Si bien es lógico y deseable que cada uno de los equipos resuelva por si mismo su manera de trabajar, desde la Tutoría se impone la condición de nombrar un coordinador del equipo y un responsable de cada uno de los nueve ejercicios que componen la totalidad del curso cuyas funciones quedan inequívocamente establecidas desde el comienzo.

El Diseño de Proyectos requiere de un análisis de la información y de aplicación de conceptos, más que una simple acumulación de datos. Cada uno de los ejercicios que conforman el Módulo de Diseño debe ser desarrollado completando una Plantilla diseñada por la Tutoría y respetando un máximo de palabras, lo que obliga al equipo a procesar y sintetizar los aportes.

El tutor debe ser capaz de acompañar el desarrollo de cada etapa, orientando cuando se requiera. Para este menester la herramienta apropiada es el "Foro de Debate" de cada uno de los ejercicios, además de comunicaciones que puedan darse por otros medios.

Las entregas deben ser consensuadas dentro de cada uno de los grupos. Nuevamente, la Tutoría actúa de manera tangencial en este sentido, exigiendo entregas previas 
parciales individuales para asegurar la participación y aporte de todos los integrantes del equipo.

Las normas de presentación deben ser precisas y estrictas. Los archivos de entrega deben estar nombrados de una manera preestablecida.

Se requiere arribar a un único proyecto final y presentarlo.

El noveno y último ejercicio consiste en una presentación del proyecto diseñado en el transcurso de la totalidad del Módulo de Diseño.

El producto final, los productos intermedios y el proceso deben ser evaluados.

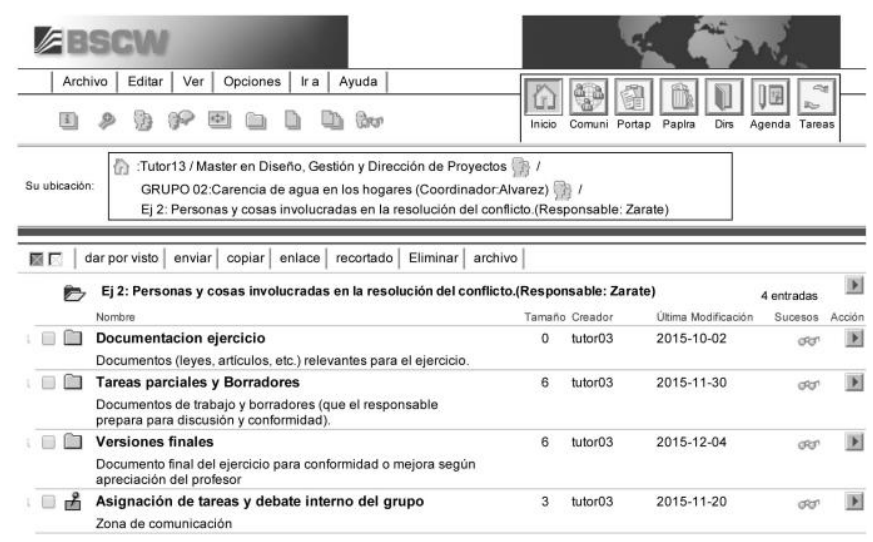

Figura 2: Carpeta de un equipo (Recuperada el 17 de febrero de 2016 de

http://bscw.ing.udep.edu.pe/bscw/bscw.cgi/9854266 )

Buscando la complementariedad entre los diferentes grupos, más allá de la evaluación por parte del docente, existen dos instancias de intercambio y corrección entre pares que se denominan Talleres Transversales. Se trata de una evaluación cruzada, de carácter individual, que tiene lugar en dos oportunidades: al finalizar el cuarto ejercicio, o sea en la mitad del proceso y otra previa a la entrega final.

En todo momento la función del docente es la de orientador y mediador cognitivo y debe buscar siempre estimular el desarrollo de las habilidades y competencias transversales que mencionábamos al comienzo para que sean internalizadas por el estudiante de posgrado y pueda, de esta forma, verá enriquecido a sí mismo como profesional y como individuo.

\section{Conclusión.}

La formación de posgrado mediante la aplicación de metodologías de enseñanza aprendizaje colaborativo, fomenta en el estudiante la adquisición de competencias transversales de trabajo en equipo, muy valoradas en el ámbito profesional.

Pese en que la aplicación del este tipo de estrategias didácticas mediadas por TICs, puede representar dificultades para algunos alumno, más que nada por deficiencias en la gestión de las comunicaciones (algo subsanable desde la entidad académica) y de cuestiones de organización personal, la mayoría de quienes se han formado de esta manera lo consideran como una experiencia positiva $y$ enriquecedora.

Como un valor agregado la adquisición de conocimientos, la práctica del trabajo colaborativo les deja la experiencia de trabajar con personas a las que no conocen personalmente y que viven en lugares distantes, lo que permite interesantes intercambios desde lo personal y lo profesional.

Otra de las habilidades que este tipo de estrategia didáctica permite desarrollar es la de la comunicación eficiente, sobre todo escrita, una de las carencias más importante a nivel de los profesionales universitarios de algunas disciplinas.

Pero, por sobre todas las cosas, se desarrollo la capacidad de autogestionar el conocimiento y a valorar el trabajo de los demás como complementario del propio e indispensable para

\section{Referencias}

Ángel,I. y Cano, L. (2011). Experiencia de un trabajo colaborativo con estudiantes y docentes de diferentes países mediado por las tecnologías de la información y la comunicación: Proyecto colaborativo interuniversitario, capítulo Colombia. Revista Q, 6 (11). 1 - 20. Recuperado el 21 de enero de 2016 de http://dialnet.unirioja.es/servlet/articulo? codigo=3989812

Badia, A. y García, C. (2006). Incorporación de las TIC en la enseñanza y el aprendizaje basados en la elaboración colaborativa de proyectos. RUSC. Universities and Knowledge Society Journal, 3 (2). 42 - 54. Recuperado el 21 de enero de 2016 de http://www.uoc.edu/rusc/3/2/dt/esp/badia_garcia.pdf

Echazarreta, C., Prados, F., Poch, J. y Soler, J. (2009). La competencia «El trabajo colaborativo»: una oportunidad para incorporar las TIC en la didáctica universitaria. Descripción de la experiencia con la plataforma ACME. Uocpapers (8). 1 - 11. Recuperado el 23 de enero de 2016 de http://www.uoc.edu/uocpapers/8/dt/esp/echazarreta_prados_ poch_soler.pdf

FUNIBER. Plataforma BSCW. Módulo de Diseño de Proyectos. http://bscw.ing.udep.edu.pe/

Hernández, N. y Muñoz, P. (2012). Trabajo colaborativo en entornos e-learning y desarrollo de competencias transversales de trabajo en equipo: Análisis del caso del Máster en gestión de Proyectos en Cooperación Internacional, CSEU La Salle. Revista de Docencia Universitaria,10 (2),411 - 434. Recuperado el 17 de enero de 2016 de http://redu.net/redu/index.php/REDU/article/view/422

Iglesias, M., Lozano, I. y Martínez, M. (2013). La utilización de herramientas digitales en el desarrollo del aprendizaje colaborativo: análisis de una experiencia en Educación Superior. Revista de Docencia Universitaria, 11 (2). 333-351. Recuperado el 21 de enero de 2016 de file:///C:/Users/Hp/Downloads/Dialnet-

LaUtilizacionDePlataformasVirtualesParaEIDesarroll4414637\%20(1).pdf

Ramírez,E. y Rojas, R. (2014). El trabajo colaborativo como estrategia para construir conocimientos. Revista Virajes, 16 (1). 89 - 101. Recuperado el 19 de enero de 2016 de http://vip.ucaldas.edu.co/virajes/downloads/Virajes16(1)_6.pdf 
Villasana,N. y Dorrego, E. (2007). Habilidades sociales en entornos virtuales de trabajo colaborativo. RIED ,10 (2). 45-74. Recuperado el 19 de enero de 2016 de http://www.biblioteca.org.ar/libros/142126.pdf

Williams, J. H. (2008). Employee engagement: Improving anticipation in safety. Professional Safety, 53(12), 40-45.
Keller, T. E., Cusick, G. R., \& Courtney, M. E. (2007). Approaching the transition to adulthood: Distinctive profiles of adolescents aging out of the child welfare system. Social Services Review, 81, 453- 484. 